\title{
Aluminium plates with pre-formed slits subjected to blast loading
}

\author{
Henrik Granum ${ }^{1, *}$, Vegard Aune ${ }^{1,2}$, Tore Børvik ${ }^{1,2}$, and Odd Sture Hopperstad ${ }^{1,2}$ \\ ${ }^{1}$ Structural Impact Laboratory (SIMLab), Department of Structural Engineering, Norwegian University of Science and Technology \\ (NTNU), NO-7491 Trondheim, Norway \\ ${ }^{2}$ Centre for Advanced Structural Analysis (CASA), NTNU, NO-7491 Trondheim, Norway
}

\begin{abstract}
The dynamic response of thin, perforated aluminium plates subjected to blast loading was studied both experimentally and numerically. Two different blast intensities were used and the plates were pre-cut with four horizontal and vertical slits prior to testing. The applied AA6016-T4 plates had an exposed area of $0.3 \mathrm{~m} \times 0.3 \mathrm{~m}$ and a thickness of $1.5 \mathrm{~mm}$. Special focus was placed on the dynamic response and failure characteristics of the plates. Uniaxial tensile tests were conducted in three different directions to determine the material behaviour and material parameters were found by inverse modelling using the optimization tool LSOPT. Finally, numerical simulations were performed in the finite element code Abaqus/Explicit where the plates were uniformly loaded with time-dependent pressure histories from similar tests on massive plates. The material behaviour was assumed to follow the $\mathrm{J} 2$ flow theory of plasticity and an uncoupled damage model was used in combination with element erosion to predict material failure. The numerical results were in good agreement with the experimental observations and predicted both the dynamic response and the complete tearing of the centre part of the plates.
\end{abstract}

\section{Introduction}

Plates subjected to blast loading have been studied for several decades and the dynamic response is well documented (see e.g. [1]). However, when introducing perforations in the plate, the dynamic response and failure mode may change, and the current literature contains limited research on this. The perforations may be a consequence of combined blast and fragmentation events or pre-formed holes to imitate a facade. Ductile failure in blast-loaded plates without perforations typically results in complete tearing along the boundary [2]. This failure mode has been simulated with good accuracy in previous studies. By introducing pre-formed holes in the plate, studies have shown that cracks may initiate at and propagate from the perforations, resulting in a more complex failure pattern. Aune et al. [3] conducted experiments on steel plates with four pre-formed holes subjected to blast loading. It was observed that cracks initiated at the extremities of the holes and notable crack growth was seen along the diagonals. The numerical simulations proved to be mesh dependent and shell elements with characteristic lengths similar to the plate thickness were needed to predict the observed failure pattern. $\mathrm{Li}$ et al. [4] conducted experiments on steel plates with circular, square and diamond shaped preformed holes to study the dynamic response. It was reported that diamond shaped holes had the lowest capacity and failure occurred by cracks propagating between the holes. Rakvåg et al. [5] also conducted experiments on steel plates with pre-formed holes subjected to pressure

\footnotetext{
*e-mail: henrik.granum@ntnu.no
}

pulse loading. The applied load was not sufficient to initiate crack propagation, but localization of plastic deformation was observed at the extremities.

This study is inspired by the perforated geometries used by Rakvåg et al. [5], where the pre-formed slits displayed the largest value of equivalent plastic strain in the simulations. It was assumed that these perforations were suited for initiating and propagating cracks when loading the plates by a blast pressure. In the numerical simulations, the constitutive model was kept relatively simple and only a single uniaxial tensile test was used to calibrate the material parameters. A refined mesh was used to capture the plastic behaviour and the ductile failure of the plate. The boundary conditions of the plate were modelled in a simplified manner and the load was applied as a pressure load with the intention to investigate the performance of an uncoupled blast-load approach.

\section{Experimental work}

\subsection{Material tests}

The $1.5 \mathrm{~mm}$ thick plates were cut from a rolled and heat treated sheet of aluminium alloy AA6016-T4 produced by Hydro Aluminium. Rolled, heat treatable alloys as the AA6016 are popular for use in automotive skin sheets [6], and have qualities such as good formability in solid solution combined with high strength when artificially aged. The plates were received with dimensions $625 \mathrm{~mm} \times 625$ $\mathrm{mm} \times 1.5 \mathrm{~mm}$ and the chemical composition of the alloy is given in Table 1. Due to the natural ageing, which is a continuous process in the $\mathrm{T} 4$ temper, the strength and work 
hardening behaviour of the material are affected by roomtemperature storage. Both the material tests and the shock tube tests were therefore conducted with as little time difference as possible.

Tensile tests on specimens taken from the plate material were conducted in three directions with respect to the rolling direction; $0^{\circ}, 45^{\circ}$ and $90^{\circ}$ with two repetitions for each direction. The dog-bone specimens had a gauge length of $70 \mathrm{~mm}$, an initial thickness of $1.5 \mathrm{~mm}$ and were all cut out from the same plate. An Instron 5982 series testing machine with a $250 \mathrm{kN}$ load cell was used with a crosshead velocity of $2.1 \mathrm{~mm} / \mathrm{min}$, which corresponds to an initial strain rate of $5 \times 10^{-4} \mathrm{~s}^{-1}$. The axial force was measured by the load cell at $1 \mathrm{~Hz}$ while 2D-DIC was used to track displacements using a virtual extensometer of 50 $\mathrm{mm}$ within an in-house DIC code. The 2D-DIC method used in this study is presented in detail in Refs. [7, 8]. To prepare the specimen for DIC, a speckle pattern was spray-painted on the gauge section and a camera operating at 1 frames per second oriented perpendicularly to the specimen captured the pictures. Representative forcedisplacement curves from the three directions and the optimized finite element analysis (FEA) of the dog-bone specimen are shown in Figure 1.

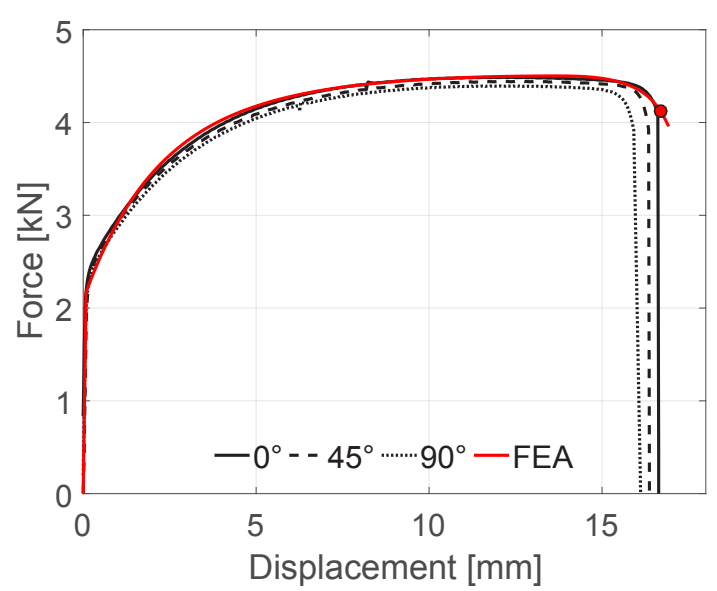

Fig. 1: Typical force-displacement curves from the tests compared to the FEA. The circle denotes the assumed point of failure used to calibrate $W_{C}$.

In addition, the assumed point of failure based on the DIC analysis is denoted with a circle. The repeated tests in each direction showed good repeatability and are not displayed. The curves reveal that the material is nearly isotropic with respect to flow stress while there is a slight difference in elongation at failure.

\subsection{Shock tube testing}

The component tests were conducted in the SIMLab Shock Tube Facility (SSTF) at NTNU. The SSTF is presented in detail in Ref. [9] and only a brief presentation will be given here. Conducting blast tests in the SSTF, as opposed to free-field airblast tests which use high explosives, gives a more controllable and accessible setup to study the dynamic response of blast-loaded structures. The SSTF consists of three main parts: a high-pressure chamber (denoted driver in Figure 2), a low-pressure chamber (denoted driven in Figure 2) and the firing section. An illustration of the experimental setup of the SSTF is shown in Figure 2, where also important dimensions are presented. The firing section is separating the driver section from the driven section with several intermediate chambers separated by diaphragms. This creates a step-wise pressure gradient between the driver and driven sections as the driver and firing sections are filled with compressed air. The shock wave is initiated by instantaneous venting of the firing section, resulting in rupture of the diaphragms which generates a shock wave travelling into the driven section. Simultaneously, rarefaction waves expand in the opposite direction towards the end of the driver section. Keeping the length of the driver section short compared to the driven section has the advantage that the rarefaction waves catch up with the shock wave, resulting in pressure-time histories similar to that in free-field airblast explosions. The planar shock waves impact the test specimen which is mounted at the end of the driven section with twelve M24 bolts in an attempt to achieve fixed boundary conditions. The exposed area of the plate corresponds to the internal cross-section of the tube in the driven section which is $0.3 \mathrm{~m} \times 0.3 \mathrm{~m}$. Upstream the driven section, two pressure sensors denoted Sensor 1 (0.245 $\mathrm{m}$ from the test specimen) and Sensor 2 ( $0.345 \mathrm{~m}$ from the test specimen) are flush mounted to obtain pressure-time histories close to the plate during a test (see Figure 2). A dump tank with windows on each side is located at the end of the driven section, covering the test specimen. Aluminium inserts were used to obtain the desired driver length of $0.77 \mathrm{~m}$, while the combined length of the firing and driven section is $16.20 \mathrm{~m}$.

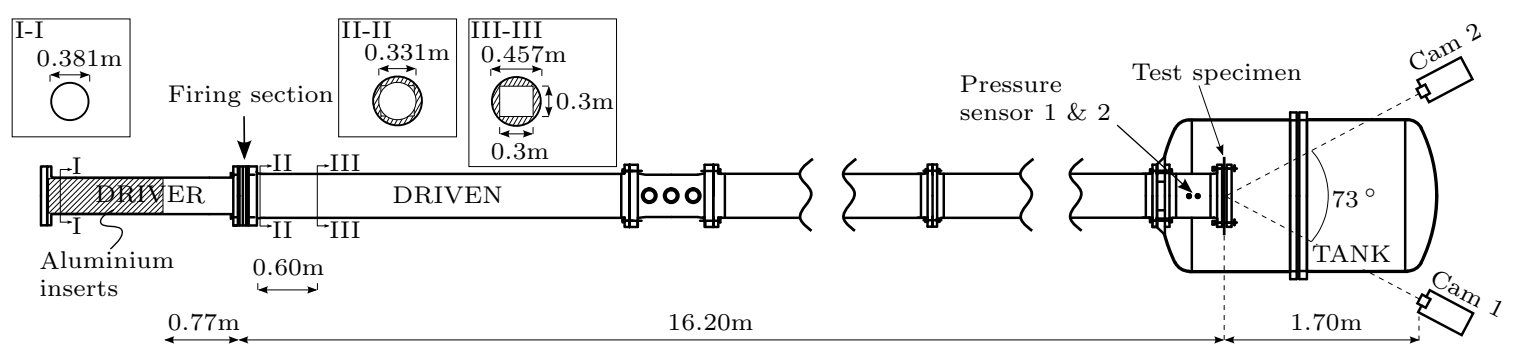

Fig. 2: Illustration of the experimental setup of the SIMLab Shock Tube Facility (STFF) [3]. 
Table 1. Chemical composition of AA6016-T4 in wt-\%.

\begin{tabular}{ccccccccc}
\hline $\mathrm{Si}$ & $\mathrm{Mg}$ & $\mathrm{Fe}$ & $\mathrm{Mn}$ & $\mathrm{Ti}$ & $\mathrm{Zn}$ & $\mathrm{Cu}$ & $\mathrm{Cr}$ & $\mathrm{Al}$ \\
\hline 1.316 & 0.349 & 0.1617 & 0.0702 & 0.0175 & 0.0084 & 0.081 & 0.0025 & Balance \\
\hline
\end{tabular}

The geometry of the perforated plate and the clamping frame is shown in Figure 3. The four slits were cut with wire erosion resulting in a width of approximately $0.1 \mathrm{~mm}$. They were placed according to Figure 3, giving double symmetry along the vertical and horizontal axes. Two blast tests were conducted at $1.0 \mathrm{MPa}$ and $1.5 \mathrm{MPa}$ initial firing pressure in the driver, denoted P-1 and P-2 hereafter, respectively.

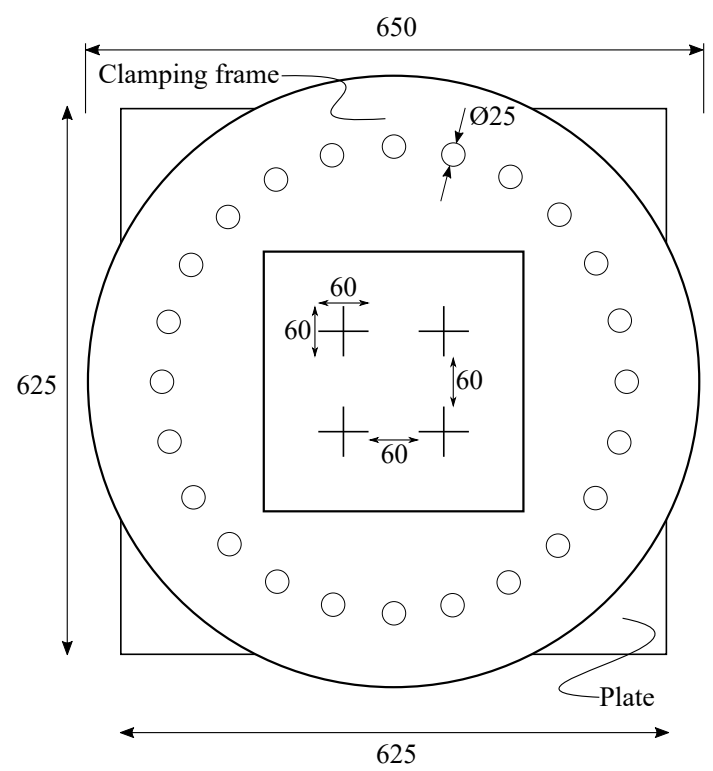

Fig. 3: Geometry of the perforated plate and clamping plate. All measures in $\mathrm{mm}$.

A typical pressure-time history from a blast load is given in Figure 4. The blast load is recognized by the instantaneous change in overpressure from zero to peak pressure $P_{\max }$ at time $t_{\mathrm{a}}$. The pressure then decays exponentially, and after a time period denoted $t_{\mathrm{d}+}$ the pressure is back at ambient level. This part of the pressure-time curve is denoted the positive phase and is followed by a negative phase. The negative phase is a result of the momentum of air which generates an overexpansion so that the absolute pressure at the tail of the blast wave falls below atmospheric pressure, resulting in a reverse flow back to the explosion centre. The negative phase occurs outside the time window of interest in these experiments, and this study will therefore only focus on the positive phase of the blast loading. To model this, the modified Friedlander equation [9] is used, given as

$$
P_{\mathrm{r}}(t)=P_{\max }\left(1-\frac{t-t_{\mathrm{a}}}{t_{\mathrm{d}+}}\right) \exp \left(\frac{-b\left(t-t_{\mathrm{a}}\right)}{t_{\mathrm{d}+}}\right)
$$

where $t$ is time and $b$ is a coefficient governing the decay of the pressure from $P_{\max }$.

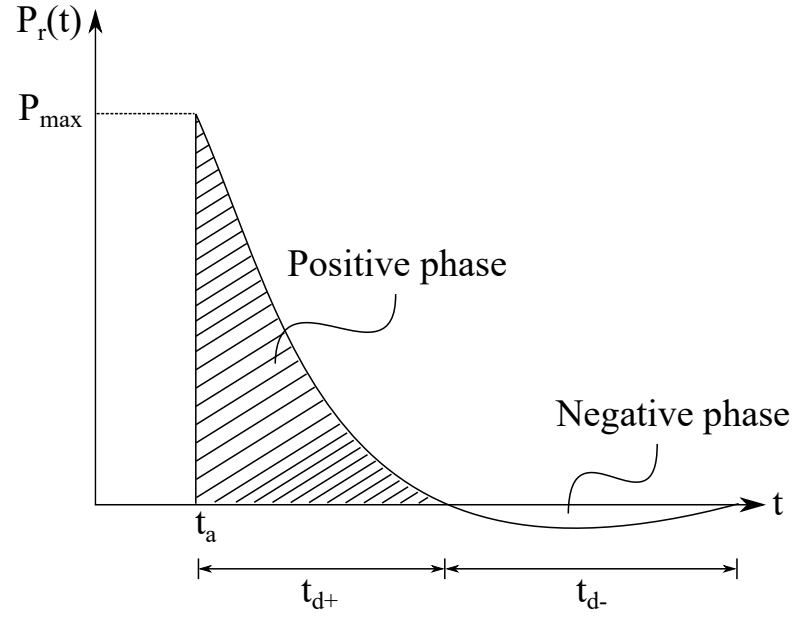

Fig. 4: Pressure-time history of a typical blast load.

The tests P-1 and P-2 are compared to tests with equivalent firing pressure on a massive plate [9] in Table 2. The incident wave parameters are taken from Sensor 1, 0.245 $\mathrm{m}$ upstream the test specimen in Figure 2. It is assumed that the deviation in maximum pressure $\Delta P_{\mathrm{so} \text {,max }}$ of the incident wave between the massive plate experiments and the corresponding tests on plates with pre-formed holes is negligible. The mack number of the shock wave $M_{\mathrm{s}}$ between these experiments is also similar. To determine the pressure load to be applied in the numerical model, an uncoupled approach is chosen which means that the pressuretime history from the massive plate tests in Ref. [9] is used. In these tests, pressure sensors were mounted directly on the plate and Eq. (1) was used to obtain idealized pressure profiles. By using this method, FSI effects are omitted which results in an overestimation of the pressure-time history. This is due to the motion of the plate which alters the pressure at its surface. An alternative is to perform coupled FSI analyses, where the shock wave is simulated as well as the structure. However, this is beyond the scope of this study.

\section{Numerical simulations}

\subsection{Constitutive modelling}

A modified Johnson-Cook (MJC) [10] constitutive relation was chosen to represent the material behaviour in the FE model. As the material was found nearly isotropic, the von Mises yield criterion was employed in combination with the associated flow rule and isotropic hardening. The constitutive relation is expressed as 
Table 2. Comparison of incident wave parameters between tests on massive and deformable plate, and idealized pressure histories in terms of Friedlander parameters curve fitted to the massive plate test data (R-1 and R-2) presented in Ref. [9].

\begin{tabular}{|l|ccc|cccc|}
\hline \multirow{3}{*}{ Test } & \multicolumn{3}{|c|}{$\begin{array}{c}\text { Incident wave } \\
\text { parameters }\end{array}$} & \multicolumn{4}{c|}{$\begin{array}{c}\text { Reflected wave parameters } \\
\text { (idealized to Eq. (1)) }\end{array}$} \\
\cline { 2 - 8 } & $M_{\mathrm{s}}$ & $P_{\text {so,max }}$ & $\Delta P_{\text {so,max }}$ & $P_{\mathrm{r}, \max }$ & $t_{\mathrm{d}+}$ & $b$ & $i_{\mathrm{r}+}$ \\
& $(-)$ & $(\mathrm{kPa})$ & $(\%)$ & $(\mathrm{kPa})$ & $(\mathrm{ms})$ & $(-)$ & $(\mathrm{kPa} \mathrm{ms})$ \\
\hline $\mathrm{R}-1$ & 1.50 & 161.7 & - & 446.2 & 35.4 & 1.571 & 4904.5 \\
$\mathrm{P}-1$ & 1.51 & 171.4 & 6.0 & - & - & - & - \\
\hline $\mathrm{R}-2$ & 1.63 & 219.1 & - & 606.6 & 44.1 & 2.025 & 7510.0 \\
$\mathrm{P}-2$ & 1.63 & 219.3 & 0.1 & - & - & - & - \\
\hline
\end{tabular}

$$
\sigma_{\mathrm{eq}}=\left(\sigma_{0}+\sum_{i=1}^{2} Q_{i}\left(1-\exp \left(-C_{i} p\right)\right)\right)\left(1+\dot{p}^{*}\right)^{c}\left(1-T^{* m}\right)
$$

where $\sigma_{\text {eq }}$ is the equivalent stress, $\sigma_{0}$ is the yield stress, $p$ is the equivalent plastic strain, $\dot{p}^{*}=\dot{p} / \dot{p}_{0}$ is the dimensionless plastic strain rate, $T^{*}=\left(T-T_{r}\right) /\left(T_{m}-T_{r}\right)$ is the homologous temperature and $m$ is the parameter controlling the thermal softening. The parameters $Q_{i}$ and $C_{i}$ determine hardening, $\dot{p}_{0}$ is a user defined strain rate, $T_{r}$ is the ambient temperature and $T_{m}$ is the melting temperature of the material. Adiabatic heating was included due to the dynamic nature of the tests and the change in temperature is calculated using

$$
\dot{T}=\frac{\chi}{\rho c_{p}} \sigma_{\mathrm{eq}} \dot{p}
$$

where $\rho$ is the density, $c_{p}$ is the specific heat and $\chi$ is the Taylor-Quinney coefficient.

Failure was taken care of by the criterion proposed by Cockcroft and Latham (CL) [11] which has the advantage of only requiring one uniaxial tensile test for calibration. The criterion is uncoupled from the constitutive model and reads

$$
D=\frac{W}{W_{c}}=\frac{1}{W_{c}} \int_{0}^{p}\left\langle\sigma_{1}\right\rangle \mathrm{d} p
$$

where $D$ is the damage variable, $W_{c}$ is the failure parameter and $\sigma_{1}$ is the major principal stress. $W_{c}$ is found by integrating the major principal stress in a uniaxial tensile test over the entire equivalent plastic strain path to the plastic strain at failure, and the material fails when $\mathrm{D}$ reaches unity. The Macaulay brackets imply that only positive values of the major principal stress contribute to the damage evolution. An important feature of the CL failure criterion is that it accounts for both the stress triaxiality $\sigma^{*}$ and the Lode parameter $\mu_{\sigma}$ in the expression for the major principal stress given as

$$
\sigma_{1}=\left(\sigma^{*}+\frac{3-\mu_{\sigma}}{3 \sqrt{3+\mu_{\sigma}^{2}}}\right) \sigma_{\mathrm{eq}}
$$

where the stress triaxiality and the Lode parameter are defined as

$$
\sigma^{*}=\frac{\sigma_{H}}{\sigma_{\mathrm{eq}}}, \quad \mu_{\sigma}=\frac{2 \sigma_{2}-\sigma_{1}-\sigma_{3}}{\sigma_{1}-\sigma_{3}}
$$

The principal stresses are ordered as $\sigma_{1} \geq \sigma_{2} \geq \sigma_{3}$ and $\sigma_{H}$ is the hydrostatic stress.

The hardening parameters $Q_{i}$ and $C_{i}$ were found by inverse modelling using the optimization tool LS-OPT, where an FE model of the tensile test was made and sequential analyses were run on the same model, varying the hardening parameters. The engineering stress-strain curve from a representative test was used as a target curve, and the mean-squared-error between the simulated and experimental target curve was minimized. To calibrate the CL failure criterion, the equivalent plastic strain $p$ was extracted from the element exposed to the largest plastic strain in a simulation of the tensile test. The failure strain $p_{f}$ was determined at the assumed point of failure taken from the force-displacement curve (see Figure 1). The major principal stress was then integrated over the equivalent plastic strain path to find $W_{c}$. The calibrated material parameters are presented in Table 3 . The remaining physical and material parameters are taken from the literature and are given in Table 4.

Table 3. Calibrated material parameters for the constitutive relation.

\begin{tabular}{ccccccc}
\hline$\sigma_{0}(\mathrm{MPa})$ & $Q_{1}(\mathrm{MPa})$ & $C_{1}$ & $Q_{1}(\mathrm{MPa})$ & $C_{1}$ & $\dot{p_{0}}\left(\mathrm{~s}^{-1}\right)$ & $W_{c}(\mathrm{MPa})$ \\
\hline 115.0 & 97.5 & 24.9 & 240.9 & 2.0 & $5 \times 10^{-4}$ & 199.0 \\
\hline
\end{tabular}

Table 4. Physical and material parameters for the material taken from the literature [12].

\begin{tabular}{ccccccccc}
\hline$E(\mathrm{MPa})$ & $v$ & $\rho\left(\mathrm{kg} / \mathrm{m}^{3}\right)$ & $c_{p}(\mathrm{~J} / \mathrm{kgK})$ & $\chi$ & $T_{r}(\mathrm{~K})$ & $T_{m}(\mathrm{~K})$ & $c$ & $m$ \\
\hline 70000 & 0.3 & 2700 & 910 & 0.9 & 293 & 923 & 0.001 & 1.0 \\
\hline
\end{tabular}


$\mathrm{t}=0 \mathrm{~ms}$
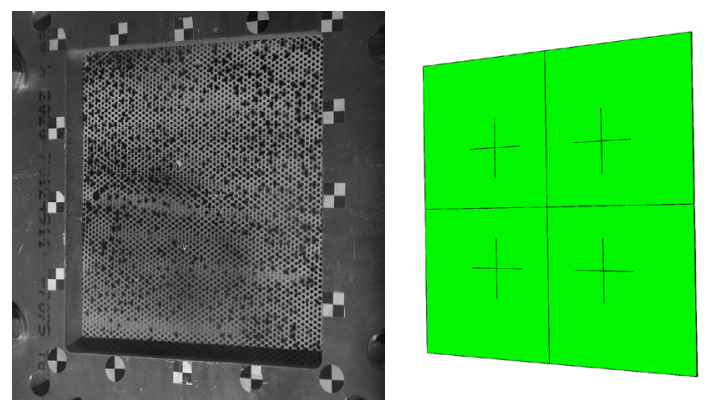

$\mathrm{t}=0.5 \mathrm{~ms}$
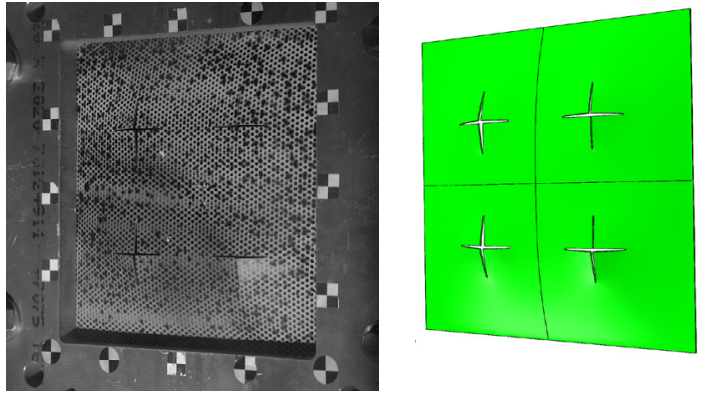

$\mathrm{t}=0.75 \mathrm{~ms}$
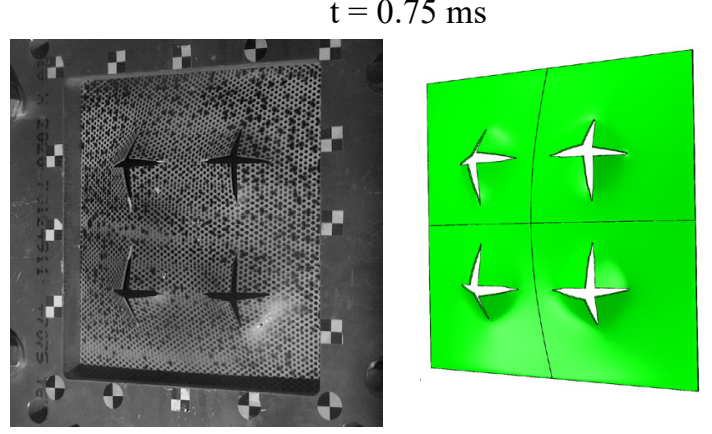

Fig. 5: Image series from experiment and simulation of the P-1 test at selected points in time.

\subsection{Finite element model}

The FE simulations were carried out using Abaqus/Explicit [13] where a quarter of the exposed area of the plate in Figure 3 was modelled. The boundary nodes were fixed against translations and rotations in an attempt to simulate the axial restraint introduced by the clamping frame. Solid cube elements with dimensions $0.5 \mathrm{~mm} \times 0.5 \mathrm{~mm} \times 0.5 \mathrm{~mm}$ with reduced integration, denoted C3D8R in Abaqus, were used, which gives three elements over the thickness and a total of 270000 elements for the quarter model. This is the same element size as employed in the simulation of the uniaxial tensile test where the CL failure criterion was calibrated. The blast load was applied as a pressure load by tabulating the idealized Friedlander curves given in Table 2. When the damage variable $D$ in Eq. (4) reaches unity in the integration point, the element was removed from the mesh.

\subsection{Results}

Image series of the numerical and experimental results from the P-1 test are compared to each other in Figure 5. Only the results from the P-1 test are shown as similar results were obtained from the P-2 test. The shock wave impacts the plate at $t=0 \mathrm{~ms}$ and the plate is given an out-of-plane displacement at $t=0.5 \mathrm{~ms}$. At $t=0.75$ $\mathrm{ms}$ a petaling deformation is seen to evolve in each of the slits, but no cracks are observed to initiate at this point in time. Cracks are then propagating from the extremities of the slits, both horizontally and vertically, resulting in the centre part of the plate being completely torn out at $t=1.0$ ms. At $t=1.5 \mathrm{~ms}$ cracks are seen to propagate from the remaining slits towards the outer boundary in the direction of the slits and the middle part of each length of the boundary folds around the clamping frame. 


\section{Concluding remarks}

Tests on AA6016-T4 plates subjected to blast loading and associated material tests have been presented. The blast tests were conducted in the SSTF at NTNU on $1.5 \mathrm{~mm}$ thick plates with four horizontal and vertical slits at two different blast intensities. Both tests experienced crack propagation and complete failure of the centre part of the plate. Material tests on specimens taken from the plate material were conducted to calibrate material parameters using 2D-DIC and the optimization tool LS-OPT. FE simulations were conducted with Abaqus/Explicit using a refined solid element mesh and the material behaviour was described by $\mathrm{J} 2$ flow theory of plasticity and an uncoupled damage model to predict failure. By comparison of the simulations and tests, good correspondence was found when predicting the crack propagation and failure pattern.

This study is part of an ongoing research project on the modelling and simulation of ductile fracture in aluminium alloys. Further studies will be carried out on the influence of slit orientation and heat treatment on the dynamic response and failure characteristics in the plates. The slit orientation may result in more complex failure patterns, while a different heat treatment will change the material's strength and ductility. These experimental results will provide a set of data which can be used to develop and evaluate new microstructure-based modelling and simulation frameworks.

The authors gratefully appreciate the financial support from NTNU and the Research Council of Norway through the FRINATEK Programme, Project No. 250553 (FractAl).

\section{References}

1. R. Rajendran, J. M. Lee, Marine Structures 22, p. 99127 (2009)

2. M. D. Olson, G.N. Nurick and J.R. Fagnan, International Journal of Impact Engineering 13, p. 279-291 (1993)

3. V. Aune, G. Valsamos, F. Casadei, M. Langseth and T. Børvik, International Journal of Impact Engineering 108, p. 27-46 (2017)

4. Y. Li, W. Wu, H. Zhu, Z. Wu and Z. Du, Engineering Failure Analysis 78, p. 122-133 (2017)

5. K .G. Rakvåg, N.J. Underwood, G.K. Schleyer, T. Børvik and O.S. Hopperstad, International Journal of Impact Engineering 53, p. 44-55 (2012)

6. O. G. Lademo, O. Engler, S. Keller, T. Berstad, K.O. Pedersen and O.S. Hopperstad, Materials and Design 30, p. 3005-3019 (2009)

7. E. Fagerholt, T. Børvik and O.S. Hopperstad, Optics and Lasers in Engineering 51, p. 299-310 (2013)

8. J. Johnsen, F. Grytten, O.S. Hopperstad and A.H. Claussen, Polymer Testing 53, p. 305-313 (2016)

9. V. Aune, E. Fagerholt, M. Langseth and T. Børvik, International Journal of Protective Structures 7(3), p. 340366 (2016)

10. T. Børvik, O.S. Hopperstad, T. Berstad and M. Langseth, European Journal of Mechanics A/Solids 20, p. 685-712 (2001)

11. M. G. Cockcroft and D.J. Latham, Journal of the Institute of Metals 96, p. 33-39 (1968)

12. J. K. Holmen, T. Børvik, O.R. Myhr, H.G. Fjær and O.S. Hopperstad, International Journal of Impact Engineering 84, p. 96-107 (2015)

13. Abaqus, Version 6.14 